\title{
SCHUR M-POWER CONVEXITY OF GENERALIZED HAMY SYMMETRIC FUNCTION
}

\section{WEN WANG AND SHIGUO YANG}

Abstract. In this paper, we investigate the Schur m-power convexity of the generalized hamy symmetric function

$$
F_{n}^{*}(\mathbf{x}, r)=\sum_{i_{1}+i_{2}+\cdots+i_{n}=r}\left(x_{1}^{i_{1}} x_{2}^{i_{2}} \cdots x_{n}^{i_{n}}\right)^{\frac{1}{r}}
$$

for $x \in \mathbb{R}_{+}^{n}$ and $r \in \mathbb{N}$ with $1 \leqslant r \leqslant n$, which generalizes some known results.

Mathematics subject classification (2010): 05E05, 26B25.

Keywords and phrases: Symmetric function, Schur m-power convexity, hamy symmetric function.

\section{REFERENCES}

[1] G. D. Anderson, M. K. Vamanamurthy, And M. Vuorinen, Generalized convexity and inequalities, J. Math. Anal. Appl., 335, 2 (2007), 1294-1308.

[2] P. S. Bullen, D. S. Mitrinovi'C And M. VAsi'C, Handbook of Means and Theirs Inequality, Kluwer Academic Publishers, Dordrecht, 2003.

[3] P. S. Bullen, A Dictionary of Inequalities, Pitman Monographs and Surveys in Pure and Applied Mathematics 97, Addison Wesley Longman Limited, 1998.

[4] Y.-M. CHU AND T.-C. SUN, The schur harmonic convexity for a class of symmetric functions, Acta mathematica Scientia, 30, B(5) (2010), 1501-1506.

[5] Y.-M. CHU AND X.-M. ZHANG, Necessary and sufficient conditions such that extended mean values are Schur-convex or Schur-concave, J. Math. Kyoto Univ., 48, 1 (2008), 229-238.

[6] A. Forcina And A. Giovagnoli, Homogeneity indices and Schur-convex functions, Statistica, 42, 4 (1982), 529-542.

[7] K.-Z. GUAN, The Hamy symmetric function and its generalization, Math. Inequal. Appl., 9 (2006), $797-805$.

[8] K.-Z. GUAN, A class of symmetric functions for multiplicatively convex function, Math. Inequal. Appl., 10 (2007), 745-753.

[9] K.-Z. GUAN, Schur-convexity of the complete symmetric function, Math. Inequal. Appl., 9, 4 (2006), 567-576.

[10] CH. Gu AND H.-N. SHI, Schur-convexity and Schur-geometric convexity of Lehmer means, Math. Prac. Theory, 39, 12 (2009), 183-188.

[11] T. HARA, M. UChTYAMA AND S. TAKAHASI, A refinement of various mean inequalities, J. Inequal. Appl., 2 (1998), 387-395.

[12] G. H. Hardy, J. E. Littlewood And G. Pólya, Some simple inequalities satisfied by convex functions, Messenger Math., 58 (1929), 145-152.

[13] F. K. Hwang And U. G. Rothblum, Partition-optimization with Schur convex sum objective functions, SIAM J. Discrete Math., 18, 3 (2004), 512-524.

[14] H. T. KU, M. C. KU AND X. M. Zhang, Inequalities for symmetric means, symmetric harmonic means and theirs applications, Bull. Austral. Math. Soc., 56 (1997), 409-420.

[15] J.-C. KuAng, Applied Inequalities (3nd ed.), Shandong Science and Technology Press, Jinan, 2004.

[16] D.-M. Li AND H.-N. SHI, Schur convexity and Schur-geometrically concavity of generalized exponent mean, J. Math. Inequal., 3, 2 (2009), 217-225. 
[17] A. W. Marshall And I. Olkin, Inequalities: Theory of Majorization and Its Applications, New York, Academic Press, 1979.

[18] F. QI, J. SÁNDOR AND S. S. DRAGOMIR, Notes on the Schur-convexity of the extended mean values, Taiwanese J. Math., 9 (2005), 411-420.

[19] H.-N. SHI, S.-H. WU AND F. QI, An alternative note on the Schur-convexity of the extended mean values, Math. Inequal. Appl., 9 (2006), 219-224.

[20] H.-N SHI AND J. ZHANG, Schur-convexity,Schur-geometric and Harmonic convexity of dual form of a class symmetric functions, J. Inequal. Appl., 2013 (2013), 295.

[21] H.-N. SHI, Y.-M. JIANG, AND W.-D. JIANG, Schur-convexity and Schur-geometrically concavity of Gini means, Comput. Math. Appl., 57, 2 (2009), 266-274.

[22] M. Shaked, J. G. Shanthikumar, And Y. L. Tong, Parametric Schur convexity and arrangement monotonicity properties of partial sums, J. Multivariate Anal., 53, 2 (1995), 293-310.

[23] B.-Y. WANG, Foundations of Majorization Inequalities (in Chinese), Beijing, Beijing Norma University Press, 1990.

[24] Z.-H. WANG AND X.-M. ZHANG, Necessary and sufficient conditions for Schur convexity and Schurgeometrically convexity of Gini means, Communications of inequalities researching, 14, 2 (2007), 193-197.

[25] W.-F. XIA AND Y.-M. ChU, The Schur convexity for a class of symmetric functions and its applications, Advances Math., 41, 4 (2012), 436-446 (in Chinese).

[26] W.-F. XIA AND Y.-M. ChU, The Schur convexity and inequalities for a class of symmetric functions, Acta Math. Sci. (Ser A), 32, A(1) (2012), 80-89 (in Chinese).

[27] W.-F. XIA, The Schur harmonic convexity of Lehmer means, Int. Math. Forum, 4, 41 (2009), 20092015.

[28] W.-F. XIA AND Y.-M. ChU, Schur-convexity for a class of symmetric functions and its applications, J. Inequal. Appl., 2009 (2009), Art. ID 493759, 15 pages.

[29] ZH.-H. YANG, Schur power comvexity of the Stolarsky means, Publ. Math. Debrecen, 80, 1-2 (2012), 43-66 DOI: 10.5486/PMD.2012.4812.

[30] ZH.-H. YANG, Schur power convexity of Gini means, Bull. Korean Math. Soc., 50, 2 (2013), 485-498.

[31] ZH.-H. YANG, Schur power comvexity of the daróczy means, Math. Inequal. Appl. 16, 3 (2013), $751-762$.

[32] ZH.-H. YANG, Necessary and sufficient conditions for Schur convexity of the two-parameter symmetric homogeneous means, Appl. Math. Sci. (Ruse), 5, 64 (2011), 3183-3190.

[33] ZH.-H. YANG, Necessary and Sufficient Conditions for Schur Geometrical Convexity of the FourParameter Homogeneous Means, Abstract and Applied Analysis, 2010, Article ID 830163, 16 pages, doi:10.1155/2010/830163

[34] ZH.-H. YANG, Schur Harmonic Convexity of Gini Means, International Math. Forum 6, 16 (2011), $747-762$.

[35] X.-M. Zhang, Geometrically-Convex Functions, Anhui Univ. Press, Hefei, 2004 (in Chinese). 